\title{
SHEAR-FREE BOUNDARY IN STOKES FLOW
}

\section{PALANIAPPAN*, S. D. NIGAM and T. AMARANATH}

School of Mathematics \& C.I.S.

University of Hyderabad

Hyderabad - 500 134, INDIA

*Department of Mathematics

Indian Institute of Science

Bangalore - 560 012, INDIA

(Received March 31, 1993)

\begin{abstract}
A theorem of Harper for axially symmetric flow past a sphere which is a stream surface, and is also shear-free, is extended to flow past a doubly-body $\mathfrak{B}$ consisting of two unequal, orthogonally intersecting spheres. Several illustrative examples are given. An analogue of Faxen's law for a double-body is observed.
\end{abstract}

KEY WORDS AND PHRASES. Shear-free, axisymmetric flow, double-body, impervious, image. bubble, drag.

1991 AMS SUBJECT CLASSIFICATION CODE(S). 76D05.

\section{INTRODUCTION.}

In Stokes' flow, no-slip circle theorems for two dimensional and sphere theorems for axisymmetric flows have been proved ([1], [2], [3], [6], [7], [9]). Recently, Harper [5] has proved the following theorem for axisymmetric flows in which a sphere is a stream surface and is also shear-free.

THEOREM. If $\psi(r, \theta)$ is the Stokes' stream function of an axially symmetric steady slow viscous flow with no singularities on the sphere $r=a$, then so is

$$
\psi_{1}(r, \theta)=\psi(r, \theta)-\left(r^{3} / a^{3}\right) \psi\left(a^{2} / r, \theta\right)
$$

$(r, \theta)$ being spherical polar coordinates and the fluid motion described by $\psi_{1}$, has the sphere $r=a$ as a shear-free stream surface for which

$$
\psi_{1}=0 \text { and } \frac{\partial}{\partial r}\left(r^{-2} \frac{\partial \psi_{1}}{\partial r}\right)=0 .
$$

The term $-\left(r^{3} / a^{3}\right) \psi\left(a^{2} / r, \theta\right)$ is defined to be the image of $\psi(r, \theta)$ in the sphere $r=a$. In the special case when $\psi(r, \theta)=\frac{U}{2} r^{2} \sin ^{2} \theta$, the theorem gives

$$
\psi_{1}(r, \theta)=\frac{U}{2} r^{2} \sin ^{2} \theta-\frac{U}{2} a r \sin ^{2} \theta .
$$

This is the stream function for the flow past a shear-free sphere, Rybczynski-Hadamard [4].

In this note, we have proved a theorem which may be considered as an extension of Harper's result when the flow is axisymmetric and takes place outside a double-body $\mathfrak{B}$, formed by two 
orthogonally intersecting unequal spheres, which is impervious and shear-free. Several illustrative examples are given and in each case the drag on the double-body is calculated. A result which is an analogue of Faxen's law [4] for a double-body is observed. We also consider a pair of spherical bubbles intersecting orthogonally and rising steadily in a viscous fluid. The drag on this system is calculated and compared with Harper's result for two equal spherical bubbles touching each other and rising in a viscous fluid.

A theorem for axisymmetric inviscid flow past a double-body has also been proved by the authors [8].

\section{THE DOUBLE-BODY $\$$ B.}

Consider a double-body $\mathfrak{B}$ (Fig. 1) formed by two unequal spheres $S_{a}$ and $S_{b}$ of radii ' $a$ ' and ' $b$ ' intersecting orthogonally, with centres $O$ and $O^{\prime}$ respectively. In the right-angled triangle $O A O^{\prime}, c^{2}=a^{2}+b^{2}$ where $O O^{\prime}=c$. In the meridian plane, join $A B$ to intersect $O O^{\prime}$ in $D$; then $O D=\frac{a^{2}}{c}, D O^{\prime}=\frac{b^{2}}{c}, D A=D B=\frac{a b}{c}$ and $D$ is inverse point of the centres $O$ and $O^{\prime}$ with respect to the spheres $S_{a}$ and $S_{b}$ respectively. Also, we have

$$
\begin{aligned}
r^{2} & =r^{\prime 2}+2 c r^{\prime} \cos \theta+c^{2}, \\
r^{\prime 2} & =r^{2}-2 c r \cos \theta+c^{2}, \\
R^{2} & =r^{2}-2 \frac{a^{2}}{c} r \cos \theta+\frac{a^{4}}{c^{2}} \\
& =r^{\prime 2}+2 \frac{b^{2}}{c} r^{\prime} \cos \theta+\frac{b^{4}}{c^{2}} .
\end{aligned}
$$

Using (2.1)-(2.4) we have

$$
\begin{array}{ll}
r^{\prime}=\frac{c}{a} R, & \text { on } r=a, \\
r=\frac{c}{b} R, & \text { on } r^{\prime}=b .
\end{array}
$$

\section{FORMULATION OF THE PROBLEM.}

We consider steady flow of an incompressible viscous fluid past a shear-free double-body $\mathfrak{B}$ formed by two unequal spheres of radii ' $a$ ' and ' $b$ ' intersecting orthogonally. Let $(r, \theta, \phi),(R, \Theta, \phi)$ and $\left(r^{\prime}, \theta^{\prime}, \phi\right)$ be the spherical polar coordinates of a point $P$ with respect to $O, D, O^{\prime}$ respectively (Fig. 1). It is well-known that in a steady, axisymmetric Stokes flow the stream function $\psi(r, \theta)$ satisfies

where

$$
D^{4} \psi=0
$$

$$
\begin{array}{cc}
D^{2}=\frac{\partial^{2}}{\partial r^{2}}+\frac{1-\eta^{2}}{r^{2}} \frac{\partial^{2}}{\partial \eta^{2}}, & \eta=\cos \theta \\
=\frac{\partial^{2}}{\partial \omega^{2}}+\frac{1}{\omega} \frac{\partial}{\partial \omega} \frac{\partial^{2}}{\partial z^{2}}, \quad \omega=r \sin \theta, \quad z=r \cos \theta
\end{array}
$$

$(\omega, z, \phi)$ being the cylindrical coordinates of $P$ with respect to $O$. Since $z$ does not occur explicitly in the operator $D^{2}$ given by equation $(3.2 \mathrm{~b})$, it is form invariant under a translation of origin along the $z$-axis. We observe that:

(A) Inversion: If $\psi(r, \theta)$ is a solution of $(3.1)$, then $(r / a)^{3} \psi\left(a^{2} / r, \theta\right)$ is also a solution.

(B) Reflection: If $\psi(\omega, z)$ is a solution of $(3.1)$, then $\psi(\omega,-z)$ is also a solution.

(C) Translation of origin: If $\psi(\omega, z)$ is a solution of $(3.1)$, then $\psi(\omega, z+h)$, where $h$ is a constant, is also a solution.

We take $z$-axis as the axis of symmetry, and $(\omega, z),\left(\omega^{\prime}, z^{\prime}\right),(\Pi, Z)$ as the cylindrical 
coordinates of $P$ with respect to $O, O^{\prime} . D$, respectively.

THEOREM. Let $\varphi_{0}(\omega, z)$ be the Stokes stream function for an axisymmetric motion of a viscous fluid in the unbounded region all of whose singularities being outside the double-body $\$$ formed by two orthogonally intersecting unequal, impervious, shear-free spheres, and suppose $\psi_{0}(\omega, z)=o\left(r^{2}\right)$ at the origin. When the shear-free boundary $\mathscr{B}$ is introduced, the stream function for the fluid external to this boundary is

$$
\begin{aligned}
\psi(\omega, z)=\psi_{0}(\omega, z) & -(r / a)^{3} \psi_{0}\left(\frac{a^{2} \omega}{r^{2}} \cdot \frac{a^{2} z}{r^{2}}\right) \\
& -\left(r^{\prime} / b\right)^{3} \psi_{0}\left(\frac{b^{2} \omega^{\prime}}{r^{\prime 2}}, c+\frac{b^{2} z^{\prime}}{r^{\prime 2}}\right) \\
& +(c R / a b)^{3} \psi_{0}\left(\frac{a^{2} b^{2}}{c^{2} R^{2}} \Pi, \frac{a^{2}\left(R^{2}-\left(b^{2} Z / c\right)\right)}{c R^{2}}\right) .
\end{aligned}
$$

The second and third terms on the right hand side of (3.3) are the images of $\psi_{0}(\omega, z)$ with respect to the spheres $S_{a}$ and $S_{b}$ respectively and the last term is the image of

in the sphere $S_{a}$.

$$
\left(-r^{\prime} / b\right)^{3} \psi_{0}\left(\frac{b^{2} \omega^{\prime}}{r^{\prime 2}}, c+\frac{b^{2} z^{\prime}}{r^{\prime 2}}\right)
$$

PROOF. The conditions to be satisfied by the function $\psi$ given in (3.3) are that

(i) the perturbation terms in (3.3) viz the $2^{\text {nd }}, 3^{\text {rd }}$ and the $4^{\text {th }}$ terms must be solutions of

(ii) $\quad \psi=0=\frac{\partial}{\partial r}\left(r^{-2} \frac{\partial \psi}{\partial r}\right)$, on $S_{a}$ and $\psi=0=\frac{\partial}{\partial r^{\prime}}\left(r^{\prime-2} \frac{\partial \psi}{\partial r^{\prime}}\right)$ on $S_{b}$ thus making $\mathfrak{B}$ a stressfree stream surface;

(iii) the singularities of the perturbation terms must lie inside the double-body $\mathfrak{B}$;

(iv) the perturbation velocity must vanish as $r \rightarrow \infty$.

By virtue of (A), (B), (C), the perturbation terms in (3.3) are the solutions of (3.1) and hence condition (i) is satisfied.

It can be shown that the expression given by (3.3) satisfy the boundary conditions on $\mathfrak{B}$ and therefore (ii) is satisfied.

Further, if a singularity of $\psi_{0}(\omega, z)$ exists at $E$ outside $\mathscr{B}^{\mathrm{B}}$, then all the singularities of the perturbation terms will lie inside $\mathfrak{B}$ (see the Appendix) and condition (iii) is satisfied.

Finally, since $\psi_{0}(\omega, z)=o\left(r^{2}\right)$ at the origin, the perturbation terms in (3.3) are at most of order $o(r)$ for large $r$. Hence, the perturbation velocity tends to zero as $r \rightarrow \infty$, and the condition (iv) is also satisfied.

\section{ILLUSTRATIVE EXAMPLES.}

\section{(I) Uniform flow past $\mathfrak{B}$}

The stream function for the uniform flow along $O z$ is

$$
\psi_{0}(r, \theta)=\frac{1}{2} U r^{2} \sin ^{2} \theta
$$

When the shear-free double-body $\mathfrak{B}$ is introduced in the flow field, then the modified stream function becomes (Using (3.3))

$$
\psi(r, \theta)=\frac{1}{2} U r^{2} \sin ^{2} \theta-\frac{1}{2} U a r \sin ^{2} \theta-\frac{1}{2} U b r^{\prime} \sin ^{2} \theta^{\prime}+\frac{1}{2} U \frac{a b}{c} R \sin ^{2} \Theta
$$

Thus the image system of uniform flow at infinity consists of three Stokeslets of strengths 
$4 \pi \mu U a, 4 \pi \mu U b,-4 \pi \mu U \frac{a b}{c}$ located at $O, O^{\prime}$, and $D$ respectively. The drag on the double-body in this case is found to be

$$
\begin{gathered}
\boldsymbol{F}=4 \pi \mu U\left(a+b-\frac{a b}{c}\right) \widehat{\boldsymbol{k}} \\
=4 \pi \mu\left(\left[a \boldsymbol{q}_{0}\right]_{\boldsymbol{O}}+\left[b \boldsymbol{q}_{0}\right]_{\boldsymbol{O}^{\prime}}-\left[\frac{[b \boldsymbol{b}}{c} \boldsymbol{q}_{\mathbf{0}}\right]_{\boldsymbol{l}}\right)
\end{gathered}
$$

where $q_{0}$ represents the velocity of the undisturbed flow and the subscripts outside the square brackets indicate the evaluation at those points respectively.

Putting $b=0$ in (4.2), we get the drag on a single shear-free sphere.

(II). Quadratic flow

In this case

$$
\psi_{0}(r, \theta)=r^{3} \sin ^{2} \theta \cos \theta
$$

and by applying the theorem we obtain

$$
\begin{gathered}
\psi(r, \theta)=r^{3} \sin ^{2} \theta \cos \theta-a^{3} \sin ^{2} \theta \cos \theta-b^{3} \sin ^{2} \theta^{\prime} \cos \theta^{\prime} \\
-b c r^{\prime} \sin ^{2} \theta^{\prime}+\frac{a^{3} b^{3}}{c^{3}} \sin ^{2} \Theta \cos \Theta \\
+\frac{a^{3} b}{c^{2}} R \sin ^{2} \Theta
\end{gathered}
$$

The image system consists of a Stokes-doublet at $O$; a Stokeslet and a Stokes-doublet at $O^{\prime}$; a Stokeslet and a Stokes-doublet at $D$. The drag is given by

$$
\begin{aligned}
\boldsymbol{F} & =8 \pi \mu \frac{b\left(a^{3}-c^{3}\right)}{c^{2}} \widehat{\boldsymbol{k}} \\
& =4 \pi \mu\left(\left[a \boldsymbol{q}_{0}\right]_{\boldsymbol{O}}+\left[b \boldsymbol{q}_{0}\right]_{\boldsymbol{O}^{\prime}}-\left[\frac{a b}{c} \boldsymbol{q}_{0}\right]_{\boldsymbol{D}}\right) .
\end{aligned}
$$

Putting $b=0$, the drag on a single shear-free sphere $r=a$ with centre at $O$ is zero, while putting $a=0, F=-8 \pi \mu b c$.

(III) Stokeslet outside B

Consider a Stokeslet of strength $F_{3} / 8 \pi \mu$, located at $E(0,0,-d)$ outside $\mathfrak{B}$ on the axis of symmetry. The stream function due to the Stokeslet in an unbounded fluid is

$$
\psi_{0}(r, \theta)=\frac{F_{3}}{8 \pi \mu} r_{1} \sin ^{2} \theta_{1}
$$

where $\left(r_{1}, \theta_{1}, \phi\right)$ are the polar coordinates of $P$ with $E$ as the origin (Fig. 1). Using the theorem, we obtain

$$
\begin{gathered}
\psi(r, \theta)=\frac{F_{3}}{8 \pi \mu}\left[r_{1} \sin ^{2} \theta_{1}-\frac{a}{d} r_{2} \sin ^{2} \theta_{2}-\frac{b}{(c+d)} r_{3} \sin ^{2} \theta_{3}\right. \\
\left.-\frac{a b}{\left(a^{2}+c d\right)} r_{4} \sin ^{2} \theta_{4}\right] .
\end{gathered}
$$

where $\left(r_{2}, \theta_{2}, \phi\right)$ and $\left(r,{ }_{3}, \theta_{3}, \phi\right)$ are the spherical polar coordinates of $P$ with $E_{a}$ and $E_{b}$ as origin, which are the inverse points of $E$ with respect to $S_{a}$ and $S_{b}$ respectively and $\left(r_{4}, \theta_{4}, \phi\right)$ are the spherical polar coordinates of $P$ with respect to $E_{b a}$; the inverse point of $E_{b}$ in the sphere $S_{a}$. The image system consists of three Stokeslets of strengths $-\frac{F_{3}}{8 \pi \mu} \frac{a}{d},-\frac{F_{3}}{8 \pi \mu} \frac{b}{(c+d)}, \frac{F_{3}}{8 \pi \mu} \frac{a b}{\left(a^{2}+c d\right)}$ located at $E_{a}, E_{b}$ and $E_{b a}$ respectively inside $\mathfrak{B}$. The drag on $\mathscr{B}$ is found to be

$$
\boldsymbol{F}=F_{3}\left(\frac{a}{d}+\frac{b}{(c+d)}-\frac{a b}{\left(a^{2}+c d\right)}\right) \hat{k}
$$




$$
=4 \pi \mu\left(\left[a \boldsymbol{q}_{0}\right]_{\boldsymbol{O}}+\left[b \boldsymbol{q}_{0}\right]_{\boldsymbol{O}^{\prime}}-\left[\frac{a b}{C} \boldsymbol{q}_{0}\right]_{\boldsymbol{D}}\right) .
$$

\section{(IV) Potential-Doublet outside \$B}

Consider a potential-doublet of strength $\alpha$ located at $E(0,0,-d)$ outside $\mathfrak{B}$ on the axis of symmetry. The stream function due to a potential-doublet in an unbounded fluid is

$$
\psi_{0}(r, \theta)=\alpha \frac{\sin ^{2} \theta_{1}}{r_{1}} .
$$

Applying the theorem we get

$$
\begin{gathered}
\psi(r, \theta)=\alpha\left[\frac{\sin ^{2} \theta_{1}}{r_{1}}-\frac{a}{d^{3}}\left(r_{2}-2 \frac{a^{2}}{d} \cos \theta_{2}+\frac{a^{4}}{d^{2} r_{2}}\right) \sin ^{2} \theta_{2}\right. \\
-\frac{b}{(c+d)^{3}}\left(r_{3}-\frac{2 b^{2}}{c+d} \cos \theta_{3}+\frac{b^{4}}{(c+d)^{2} r_{3}}\right) \sin ^{2} \theta_{3} \\
+\frac{a b}{\left(a^{2}+c d\right)^{3}}\left(c^{2} r_{4}+\frac{2 a^{2} b^{2} c}{\left(a^{2}+c d\right)} \cos \theta_{4}\right. \\
\left.\left.+\frac{a^{4} b^{4}}{\left(a^{2}+c d\right)^{2} r_{4}}\right) \sin ^{2} \theta_{4}\right] .
\end{gathered}
$$

The image system consists of:

(i) three Stokeslets of strengths $-\alpha \frac{a}{d^{3}},-\alpha \frac{b}{(c+d)^{3}}, \alpha \frac{a b c^{2}}{\left(a^{2}+c d\right)^{3}}$ located at $E_{a}, E_{b}, E_{b a}$;

(ii) three Stokes-doublets of strengths $2 \alpha \frac{a^{3}}{d^{4}}, 2 \alpha \frac{b^{3}}{(c+d)^{4}}, 2 \alpha \frac{a^{3} b^{3} c}{\left(a^{2}+c d\right)^{3}}$ located at $E_{a}, E_{b}, E_{b a}$;

(iii) three potential-doublets of strengths $-\alpha \frac{a^{5}}{d^{5}},-\alpha \frac{b^{5}}{(c+d)^{5}}, \alpha \frac{a^{5} b^{5}}{\left(a^{2}+c d\right)^{5}}$ located at $E_{a}, E_{b}, E_{b a}$ respectively.

The drag in this case is

$$
\begin{aligned}
\boldsymbol{F} & =8 \pi \mu \alpha\left(\frac{a}{d^{3}}+\frac{b}{(c+d)^{3}}+\frac{a b c^{2}}{\left(a^{2}+c d\right)^{3}}\right) \widehat{\boldsymbol{k}} \\
& =4 \pi \mu\left(\left[a \boldsymbol{q}_{0}\right]_{\boldsymbol{O}}+\left[b \boldsymbol{q}_{0}\right]_{\boldsymbol{O}^{\prime}}-\left[\frac{a b}{c} \boldsymbol{q}_{0}\right]_{\boldsymbol{D}}\right) .
\end{aligned}
$$

In the examples considered, we find that the drag on the double-body is given by

$$
\boldsymbol{F}=4 \pi \mu\left(\left[a q_{0}\right]_{\boldsymbol{O}}+\left[b \boldsymbol{q}_{0}\right]_{\boldsymbol{O}^{\prime}}-\frac{a b}{c}\left[\boldsymbol{q}_{0}\right]_{\boldsymbol{D}}\right) .
$$

where $\left[\boldsymbol{q}_{0}\right]_{\boldsymbol{O}},\left[\boldsymbol{q}_{0}\right]_{\boldsymbol{O}^{\prime}}\left[\boldsymbol{q}_{0}\right]_{\boldsymbol{D}}$ are the velocity of the undisturbed flow evaluated at $O, O^{\prime}, D$ respectively. If we put $b=0$ in (4.9), we get the drag formula for a single shear-free sphere of radius ' $a$ '. This result is an analogue of Faxen's law [4] for a double-body.

\section{APPLICATION TO RISING BUBBLES.}

We consider two equal spherical bubbles intersecting orthogonally and rising steadily with a velocity $U$ in a viscous fluid. The stream function for the motion of this double-body is obtained from (4.1) by taking $a=b$. This gives

$$
\psi(r, \theta)-\frac{1}{2} U a\left[r \sin ^{2} \theta+r^{\prime} \sin ^{2} \theta^{\prime}-\frac{1}{\sqrt{2}} R \sin ^{2} \Theta\right]
$$

The drag on the double-body is 


$$
\boldsymbol{F}=5.172 \pi \mu U a \hat{\boldsymbol{k}}
$$

The total drag for two equal bubbles touching each other and rising in a viscous fluid is given by, Harper (1983)

$$
\boldsymbol{F}=5.54 \pi \mu L^{\top} a \widehat{\boldsymbol{k}}
$$

Hence, the drag on the double-body is less than that of the total drag on touching bubbles, when they are rising in a viscous fluid which is at rest, otherwise.

\section{APPENDIX}

As $E$ (Fig. 1) moves from $z=-\infty$ to $E^{\prime}$, the inverse point of $E$ with respect to $S_{a}$, namely $E_{a}$ moves from $O$ to $E^{\prime}$; also the inverse point of $E$ with respect to $S_{b}$, namcly $E_{b}$ moves from $O^{\prime}$ to $F$. Now the inverse point of $E_{b}$ with respect to $S_{a}$, namely $E_{b a}$ lies on the segment $D F$. Therefore, the singularities of the perturbation terms in (3.3) lie inside $\mathfrak{B}$.

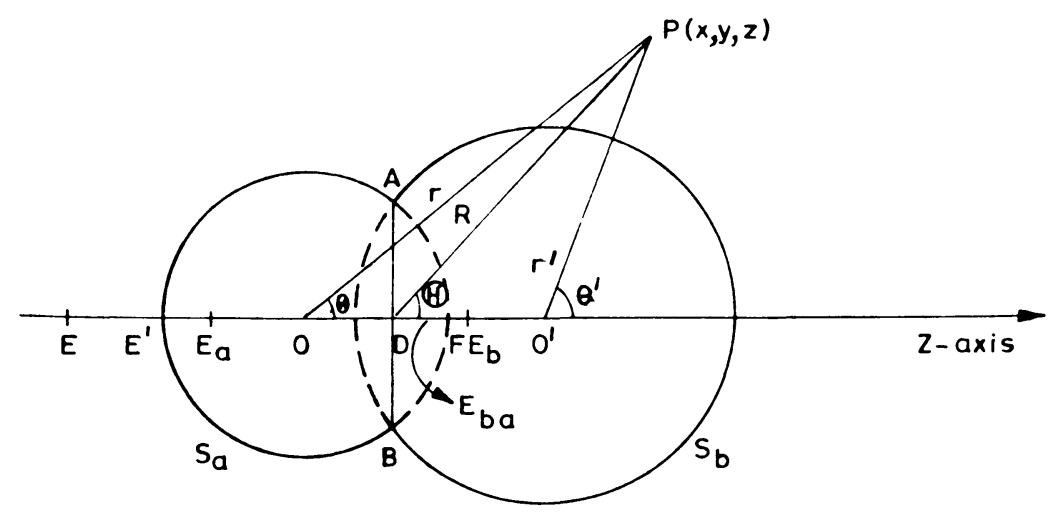

FIGURE 9. THE DOUBLE BODY $\beta$

\section{REFERENCES}

1. AVUDAINAYAGAM, A. \& JOTHIRAM, B., A circle theorem for plane Stokes flows, QJMAM 41, pt. 3 (1988), 383-393.

2. COLLINS, W.D., A note on Stokes stream function for the slow steady motion of a viscous fluid before plane and spherical boundaries, Mathematika 1 (1954), 125-130.

3. COLLINS, W.D., Note on a sphere theorem for the axisymmetric Stokes flow of a viscous fluid, Mathematika 5 (1958), 118-121.

4. HAPPEL, J. \& BRENNER, H., Low Reynolds Number Hydrodynamıcs, Prentice-Hall, Englewood Cliffs, 1965.

5. HARPER, J.F., Axisymmetric Stokes flow images in spherical free surfaces with applications to rising bubbles, J. Aust. Math. Soc. Ser. B 25 (1983), 217-231.

6. HASIMOTO, H., A sphere theorem on the Stokes equation for axisymmetric viscous flow, J. Phys. Soc. Japan 7 (1956), 793-797.

7. MARTINEK, J. \& THIELMAN, H.P., Circle and sphere theorems for the biharmonic equation (interior and exterior problems), ZAMP 16 (1965), 494-501.

8. PALANIAPPAN, D.; NIGAM, S.D. \& AMARANATH, T., A theorem for inviscid flow past two orthogonally intersecting unequal spheres, J. Math. Phys. Sci. 24, no. 5 (1990), 289-295.

9. SEN, S.K., Circle theorems for steady Stokes flow, ZAMP 40 (1989), 139-145. 


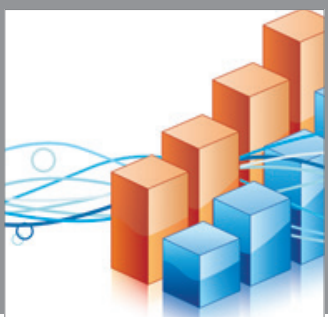

Advances in

Operations Research

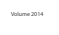

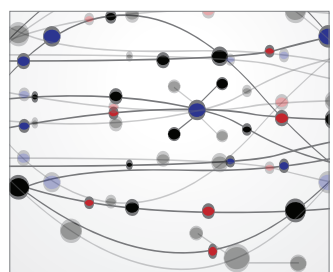

\section{The Scientific} World Journal
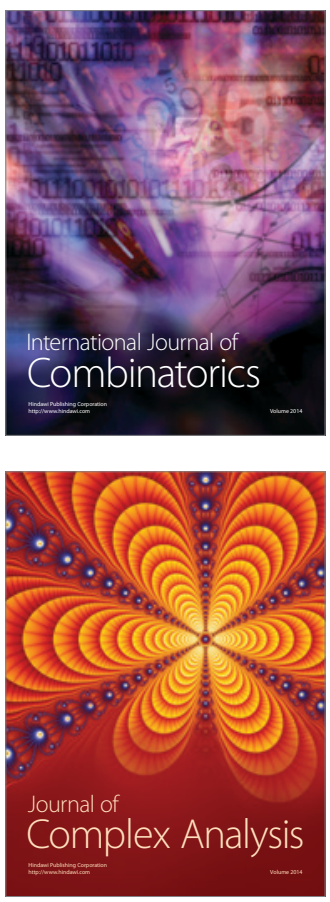

International Journal of

Mathematics and

Mathematical

Sciences
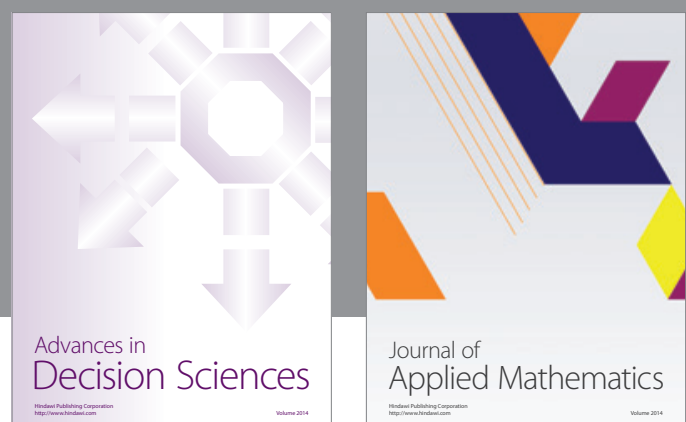

Journal of

Applied Mathematics
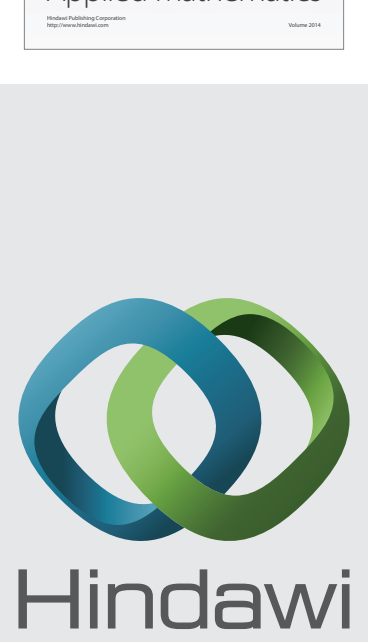

Submit your manuscripts at http://www.hindawi.com
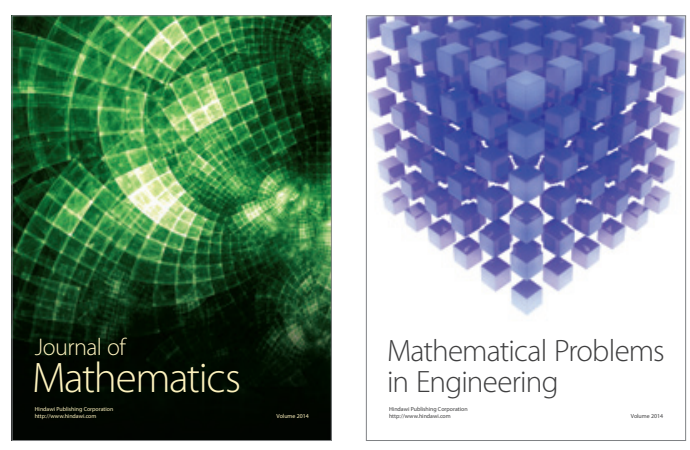

Mathematical Problems in Engineering
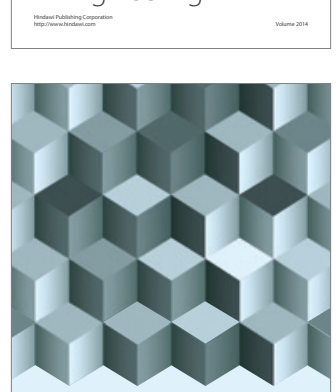

Journal of

Function Spaces
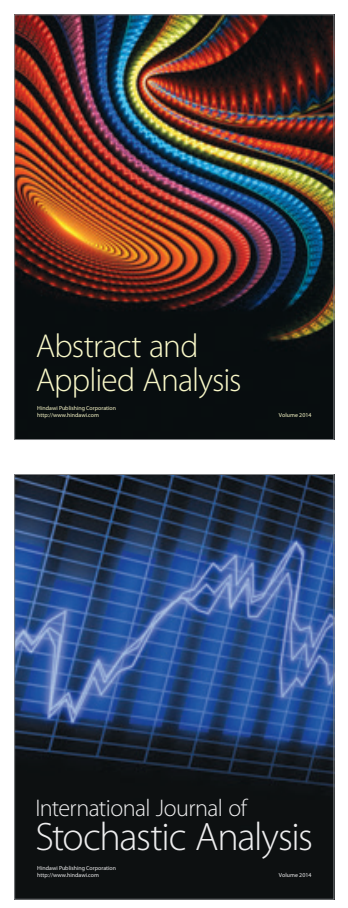

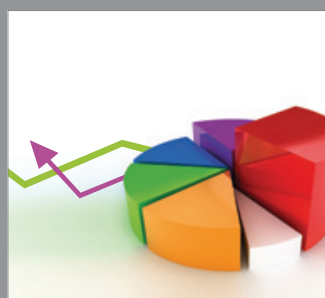

ournal of

Probability and Statistics

Promensencen
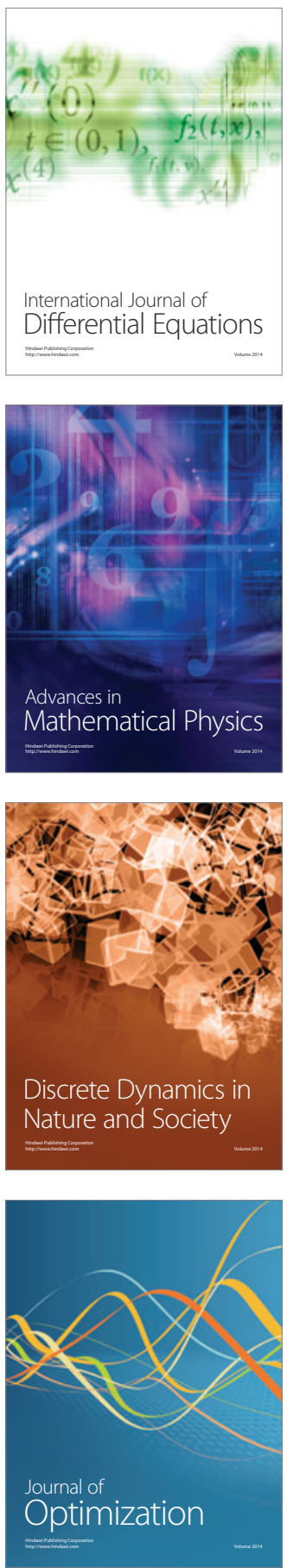